\title{
Bacterias patógenas multidrogoresistentes aisladas en estetoscopios de médicos en un hospital de nivel III
}

\author{
Multi-drug resistant bacteria isolated from medical stethoscopes in a level III hospital \\ José Oliva-Menacho ${ }^{1,2}$, José Oliva-Candela², Marco Garcia-Hjarles ${ }^{1,2}$ \\ RESUMEN
}

Objetivos: Determinar el perfil de susceptibilidad de bacterias patógenas aisladas en estetoscopios usados por médicos de un hospital de nivel III en Lima, Perú. Material y métodos: Estudio observacional, descriptivo y transversal, realizado en el Hospital Nacional Arzobispo Loayza. Ciento veintitrés bacterias aisladas se guardaron congeladas a $-20^{\circ} \mathrm{C}$. Posteriormente se sembró en placas de agar TSA, manitol salado y se procedió a incubar 24 horas a $37^{\circ} \mathrm{C}$. Se preparó una suspensión ajustada a 0,5 McFarland de turbidez, se procedió a inocular en placas de agar Mueller - Hinton (disco difusión); se determinó concentración mínima inhibitoria (MIC) en algunos casos y se utilizó antibióticos según la CLSI (Clinical and Laboratory Standards Institute). Resultados: Todas las cepas de Staphylococcus spp. coagulasa negativa, presentaron sensibilidad a ERI, DA, CIP, NF, LZD, VAN (100\%); tres bacterias dieron resistencia a PEN, OXA, FOX (2,8\%). Las cinco cepas de Staphylococcus aureus aisladas, presentaron sensibilidad a GE, AK, NF, LZD (100\%); sin embargo, una cepa de Staphylococcus aureus presentó resistencia a FOX, VAN (20\%). Las dos cepas de Acinetobacter spp. presentaron sensibilidad a IMI, MER, COL (100\%). De las cuatro cepas de Pseudomonas aeruginosa, dos presentaron resistencia a FEP, IMI, MER (50\%). La cepa de Escherichia coli presentó resistencia a IMI, MER. Conclusiones: Se tipificaron cepas de Staphylococcus coagulasa negativa y Staphylococcus aureus con resistencia a meticilina y vancomicina; Pseudomonas aeruginosa con resistencia a carbapenemasas y enterobacterias resistentes a cefalosporinas.

PALABRAS CLAVE: Estetoscopios, farmacorresistencia bacteriana, infección hospitalaria, Perú. (Fuente: DeCS BIREME).

\section{SUMMARY}

Objectives: To determine the susceptibility profile of pathogenic bacteria isolated from medical stethoscopes in a level II hospital in Lima. Methods: Cross-sectional study carried-out in Hospital Nacional Arzobispo Loayza. One hundred and twenty-three bacteria were stored at -20C to be cultured in TSA agar and salt mannitol agar to be further incubated for 24 hours at 37C. A suspension adjusted at 0,5 McFarland turbidity was done to be cultured at Mueller - Hinton agar to determine minimal inhibitory concentration (CIM) following CLSI (Clinical and Laboratory Standard Institute) recommendations. Results: All coagulase-negative Staphylococcus spp. were susceptible to ERI, DA, CIP, NF, LZD, VAN (100\%); three strains were resistant to PEN, OXA, FOX (2,8\%). All five Staphylococcus aureus strains were susceptible to GE, AK, NF, LZD (100\%); however, one strain was resistant to FOX, VAN (20\%). The two Acinetobacter spp. strains were susceptible to IMI, MER, COL (100\%). Two out of four strains of Pseudomonas were resistant to IMI, MER. Conclusions: The study reports strains of methicillin

\footnotetext{
Facultad de Medicina Alberto Hurtado, Universidad Peruana Cayetano Heredia. Lima, Perú.

Hospital Nacional Arzobispo Loayza. Lima, Perú.
} 
resistant coagulase negative Staphylococcus and Staphylococcus aureus; carbapenem resistant Pseudomonas aeruginosa, and cephalosporin resistant enterobacteria.

KEYWORDS: Stethoscopes, bacterial resistance, hospital infection, Peru. (Source: MeSH NLM).

\section{INTRODUCCIÓN}

Los estetoscopios contaminados con bacterias patógenas pueden generar infecciones nosocomiales, un problema de salud a nivel global por la pérdida de vidas humanas y el impacto económico, tanto para las familias, como para el sistema hospitalario. Las tasas de infecciones nosocomiales son mayores en los países en desarrollo (1-4).

Se ha descrito a las superficies inanimadas de los hospitales como fuente de infección intrahospitalaria (5-7); existe evidencia de transmisión indirecta por saludo con manos o guantes del trabajador contaminado cuando va tocando las superficies inanimadas o después de que examinan a los pacientes, o cuando un paciente entra en contacto directo con una superficie contaminada. Los patógenos que contaminan las superficies ambientales y equipos médicos, incluyen MRSA (Staphylococcus aureus resistente a la Meticilina), VRE (Enterococo resistente a la Vancomicina), Clostridium difficile, Acinetobacter spp y norovirus (8-9).

El estetoscopio entra en contacto directo con el paciente, y generalmente es transportado en el bolsillo del guardapolvo o cuello, sin muchos cuidados de asepsia. El estetoscopio es un vector de infección hospitalaria, varios trabajos demuestran la contaminación del mismo por microorganismos entre ellos el Staphylococcus aureus (10-13). Sthaphylococcus aureus presenta capacidad de adquirir resistencia a los antibióticos, inicialmente a la penicilina, y actualmente a meticilina, lo que representa un problema (14).

La Red de Investigación Neonatal de los Estados Unidos halló un alto porcentaje de colonización, en estetoscopios por bacterias patógenas resistentes a antibióticos de amplio espectro (Enterobacterias resistentes a cefalosporinas de tercera generación) (15).

Un estudio realizado en el área pediátrica de un hospital universitario en Brasil, demostró que $87 \%$ de estetoscopios presentaron diafragmas contaminados; el 95\% con Staphylococcus spp y el $70 \%$ con Streptococcus spp presentaron sensibilidad a oxacilina, dos cepas de Staphylococcus presentaron sensibilidad intermedia a vancomicina y una de ellas presentó resistencia a teicoplanina (16).

El objetivo del estudio fue determinar el perfil de susceptibilidad de bacterias patógenas aisladas en estetoscopios usados por médicos de un hospital de nivel 3 en Lima, Perú.

\section{MATERIAL Y MÉTODOS}

Estudio descriptivo, observacional y transversal, realizado en el Hospital Nacional Arzobispo Loayza entre enero y junio del 2013 (17). La muestra de estudio estuvo constituida por 123 bacterias patógenas tipificadas provenientes de hisopados del diafragma de los estetoscopios del personal médico.

Las bacterias se guardaron en viales con Tripticasa soya agar (Oxoid $\AA$, UK), como medio de crecimiento, leche descremada (sustancias lioprotectoras), y congeladas a $-20^{\circ} \mathrm{C}$ para su conservación.

Para recuperar la bacteria se procedió a inocular en caldo infusión cerebro y corazón (BD) por 24 horas a $37^{\circ} \mathrm{C}$, luego se sembró en placas de Tripticasa soya agar $\left(\right.$ Oxoid ${ }^{\circledR}$, UK), manitol salado agar $\left(\right.$ Oxoid ${ }^{\circledR}$, UK), MacConkey agar (Oxoid®, UK) y se procedió a incubar por 24 horas a $37^{\circ} \mathrm{C}$.

De al menos 5 colonias tomadas directamente de una placa de Tripticasa soya agar (Oxoid $\AA$, UK), manitol salado agar (Oxoid ${ }^{\circledR}$, UK), MacConkey agar (Oxoid®, UK) de igual tamaño y forma la cepa bacteriana, se preparó una suspensión del microorganismo en un caldo Mueller-Hinton $\left(\right.$ Merck ${ }^{\circledR}$, $\mathrm{kGaA}$ ). La suspensión fue ajustada a 0,5 McFarland de turbidez usando un espectrofotómetro. Esta suspensión contenía aproximadamente 1 a $4 \times 10^{8} \mathrm{UFC} / \mathrm{ml}$, dentro de los 15 minutos después de preparada la suspensión se procedió a inocular la placa en agar Mueller Hinton $\left(\right.$ Merck $\left.{ }^{\circledR}, \mathrm{kGaA}\right)$. Se utilizaron antibióticos según la Clinical and Laboratory Standards Institute (CLSI), y se midió el halo de sensibilidad para 
detectar la resistencia antimicrobiana; se determinó concentración inhibitoria mínima para algunas tipificaciones y sensibilidad microbiológica.

Los antibióticos que se utilizaron para determinar la susceptibilidad antimicrobiana en Staphylococcus spp. coagulasa negativa, fueron: Eritromicina (ERI) $(15 \mu \mathrm{g})$, Clindamicina (CLI) $(2 \mu \mathrm{g})$, Ciprofloxacino $(\mathrm{CIP})(5 \mu \mathrm{g})$, Nitrofurantoína (NIT) $(300 \mu \mathrm{g})$, Linezolid

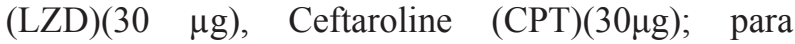
Staphylococcus aureus fueron: Oxacilina (OXA) $(1 \mu \mathrm{g})$, Amikacina (AMK) $(30 \mu \mathrm{g})$, Gentamicina (GEN) $(10 \mu \mathrm{g}), \quad$ Eritromicina $(\mathrm{ERI})(15 \mu \mathrm{g}), \quad$ Clindamicina $(\mathrm{CLI})(2 \mu \mathrm{g})$, Cefoxitin $(\mathrm{FOX})(30 \mu \mathrm{g})$, Nitrofurantoina (NIT) $(300 \mu \mathrm{g})$, Ceftaroline $(\mathrm{CPT})(30 \mu \mathrm{g})$, Linezolid (LZD) $(30 \mu \mathrm{g})$; para Vancomicina (VAN) se determinó MIC $(\mu \mathrm{g} / \mathrm{ml})$; para Enterobacter aerogenes fueron: Amoxicilina/ácido clavulánico (AMC) $(20 / 10 \mu \mathrm{g})$, Ceftazidima (CAZ) $(30 \mu \mathrm{g})$, Cefepime (FEP)(30 $\mu \mathrm{g})$, Ceftriaxona (CRO) $(30 \mu \mathrm{g})$, Aztreonam (ATM) $(30 \mu \mathrm{g})$, Amikacina (AMK) $(30 \mu \mathrm{g})$, Imipenem (IPM) $(10 \mu \mathrm{g})$,

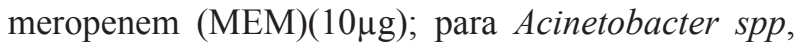
fueron: Cefotaxima (CTX) $(30 \mu \mathrm{g})$, Cefepime (FEP) $(30 \mu \mathrm{g})$, Ceftazidima (CAZ)(30 $\mu \mathrm{g})$, Gentamicina $(\mathrm{GEN})(10 \mu \mathrm{g})$, Amikacina (AMK) $(30 \mu \mathrm{g})$, Imipenem $(\mathrm{IPM})(10 \mu \mathrm{g}), \quad$ Meropenem $(\mathrm{MEM})(10 \mu \mathrm{g}), \quad$ para Pseudomonas aeruginosa, se utilizaron: Cefepime (FEP) $(30 \mu \mathrm{g})$, Ceftazidima (CAZ) $(30 \mu \mathrm{g})$, Imipenem

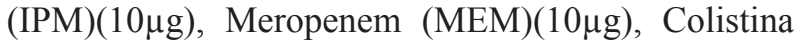
$(\mathrm{COL})(10 \mu \mathrm{g})$; para Klebsiella pneumonia fueron:

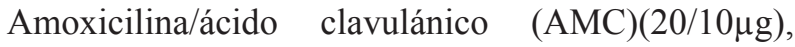
Nitrofurantoina (NIT)(300 $\mu \mathrm{g})$, Ceftazidima (CAZ) $(30 \mu \mathrm{g})$, Ceftriaxona $(\mathrm{CRO})(30 \mu \mathrm{g})$, Cefepime (FEP) $(30 \mu \mathrm{g})$, Imipenem (IPM) $(10 \mu \mathrm{g})$, Gentamicina (GEN) $(10 \mu \mathrm{g})$, Amikacina (AMK) $(30 \mu \mathrm{g})$; para Escherichia coli se utilizaron: Amoxicilina/ácido clavulanico $(\mathrm{AMC})(20 / 10 \mu \mathrm{g}), \quad$ Imipenem $\quad(\mathrm{IPM})(10 \mu \mathrm{g})$,

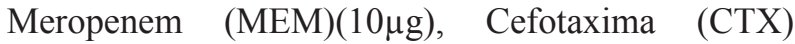
$(30 \mu \mathrm{g})$, Cefepime (FEP) $(30 \mu \mathrm{g})$, Ceftriaxona (CRO) (30 $\mu \mathrm{g})$, Ceftazidima $(\mathrm{CAZ})(30 \mu \mathrm{g})$. Las cepas de referencia utilizadas como control de calidad fueron: Escherichia coli (ATCC 25922), Staphylococcus

Tabla 1. Sensibilidad de bacterias patógenas en estetoscopios en el Hospital Nacional Arzobispo Loayza.

\begin{tabular}{|c|c|c|c|c|}
\hline \multirow[b]{2}{*}{ ANTIBIOTICOS } & \multicolumn{2}{|c|}{$\begin{array}{c}\text { Staphylococcus spp. } \\
\text { coagulasa negativa }(\mathrm{n}=106)\end{array}$} & \multicolumn{2}{|c|}{$\begin{array}{c}\text { Staphylococcus aureus } \\
(\mathrm{n}=5)\end{array}$} \\
\hline & n & $\%$ & $\mathbf{n}$ & $\%$ \\
\hline \multicolumn{5}{|l|}{ B- lactámicos } \\
\hline Penicilina G & 103 & 97,1 & 2 & 40,0 \\
\hline Oxacilina & 103 & 97,1 & 2 & 40,0 \\
\hline Cefoxitin & 103 & 97,1 & 2 & 40,0 \\
\hline Ceftaroline & 106 & 100,0 & 5 & 100,0 \\
\hline \multicolumn{5}{|l|}{ Glicopeptidos } \\
\hline Vancomicina & 106 & 100,0 & 4 & 80,0 \\
\hline \multicolumn{5}{|l|}{ Aminoglicosidos } \\
\hline Gentamicina & 103 & 97,1 & 5 & 100,0 \\
\hline Amikacina & 103 & 97,1 & 5 & 100,0 \\
\hline \multicolumn{5}{|l|}{ Macrólidos } \\
\hline Eritromicina & 106 & 100,0 & 2 & 40,0 \\
\hline \multicolumn{5}{|l|}{ Lincosamidas } \\
\hline Clindamicina & 106 & 100,0 & 5 & 100,0 \\
\hline \multicolumn{5}{|l|}{ Nitrofurantoina } \\
\hline Nitrofurantoina & 106 & 100,0 & 5 & 100,0 \\
\hline \multicolumn{5}{|l|}{ Fluroquinolonas } \\
\hline Ciprofloxacino & 106 & 100,0 & 5 & 100,0 \\
\hline \multicolumn{5}{|l|}{ Oxazolidinones } \\
\hline Linezolid & 106 & 100,0 & 5 & 100,0 \\
\hline \multicolumn{5}{|l|}{ Inhibidores de vía de folato } \\
\hline Sulfamethoxazole/Trimethoprim & 103 & 97,1 & 5 & 100,0 \\
\hline
\end{tabular}


aureus (ATCC 25923), Pseudomonas aeruginosa (ATCC27853) y Escherichia coli (ATCC 5218).

Se utilizó estadística descriptiva. Se utilizó el programa Microsoft Excel 2013 y el programa estadístico SPSS, versión 11.5 para Windows.

\section{RESULTADOS}

Los 106 aislamientos de Staphylococcus spp. coagulasa negativa, presentaron una elevada sensibilidad ERI, CLI, CIP, NIT, LZD, CPT, VAN (100\%); así mismo 103 aislamientos presentaron sensibilidad a penicilina G (PEN), OXA, FOX, GEN, AMK, sulfametoxazol/trimetoprim (SXT/TRM) (97,1\%) (Tabla1).

Los 5 aislamientos de Staphylococcus aureus, presentaron sensibilidad a GEN, AMK, NIT, CPT, LZD $(100 \%), 4$ presentaron sensibilidad a FOX $(80 \%)$, y dos presentaron sensibilidad a PEN, ERI, OXA (60\%) (Tabla 1). Un aislamiento de Staphylococcus aureus presentó resistencia a Vancomicina, (MIC $>32 \mu \mathrm{g} / \mathrm{ml}$ ).

De los cuatro aislamientos de Pseudomonas aeruginosa, dos presentaron resistencia elevada a cefuroxima (CXM), CAZ, FEP, IPM, MEM (50\%).

Los dos aislamientos de Acinetobacter spp., presentaron una elevada sensibilidad a CXM, CAZ, FEP, CIP, GEN, AMK, IPM, MEM, COL (100\%); los cuatro aislamientos de Enterobacter aerogenes, presentaron una elevada sensibilidad a CIP, NIT, GEN, AMK, IPM, MEM (100\%); tres aislamientos de Enterobacter aerogenes, presentaron resistencia a CXM, CAZ (75\%) y una, a FEP (25\%).

La cepa de Klebsiella pneumoniae presentó sensibilidad a CXM, CAZ, FEP, CIP, NIT, AMK, GEN, IPM, MEM, y la Escherichia coli aislada presentó una elevada resistencia a IPM, MEM, CAZ, y CXM.

\section{DISCUSIÓN}

La detección de Staphylococcus coagulasa negativa y Staphylococcus aureus multidrogo resistentes en este estudio de investigación es similar a lo hallado por otros autores (18-20).

El nivel de contaminación de estetoscópios por Staphylococcus aureus resistentes a la meticilina es de $17 \%$ (21); en nuestra investigación la resistencia a meticilina encontrada fue 2,8\% en Staphylococcus spp. coagulasa negativa y $20 \%$ en Staphylococcus aureus.

En este estudio se tipificó bacterias no fermentadoras resistentes a los carbapenémicos en el diafragma de los estetoscopios; en las cepas de Pseudomonas aeruginosa se evidenció resistencia a imipenem y meropenem, como ha sido descrito en otros estudios $(22,23)$.

La contaminación de estetoscopios con enterobacterias resistentes a drogas de última generación en la unidad de cuidado intensivo (24), los ha convertido en un mecanismo potencial de contaminación cruzada por microorganismos, suponiendo un riesgo para los pacientes (25-26).

En conclusión, el estetoscopio es un potencial instrumento capaz de propagar bacterias patógenas, en la investigación se tipificaron cepas de Staphylococcus coagulasa negativa y Staphylococcus aureus resistente a meticilina y a vancomicina, Pseudomonas aeruginosa con resistencia a carbapenémicos y enterobacterias resistentes a cefalosporinas.

Sin embargo, el estetoscopio es uno de los dispositivos médicos que menos se desinfectan (17); por ello, se recomienda desinfectar periódicamente los estetoscopios con desinfectantes como la clorhexidina al 4\%, alcohol etílico al 70\%, alcohol iodado, alcohol isopropilico al 70\%, para controlar la diseminación de agentes infecciosos.

\section{Declaración de financiamiento y de conflictos de interés:}

El estudio fue financiado por los autores. Los autores declaran no tener conflicto de interés.

\section{Contribuciones de autoría:}

JOM, MGH, JOC han participado en la concepción del estudio, la recolección de datos, interpretación de los datos y redacción del artículo. Todos los autores aprobaron la versión final del manuscrito.

\section{Correspondencia:}

José Oliva - Menacho.

Jr. José María Morellos 207- 402. San Miguel. Lima, Perú.

Correo electrónico: jose.oliva.m@upch.pe.

Celular: (511)990009733 


\section{REFERENCIAS BIBLIOGRÁFICAS:}

1. Allegranzi B, Bagheri S. Burden of endemic healthcare-associated infection in developing countries: systematic review and metaanalysis. Lancet. 2011; 377(9761):228- 41.

2. Baptista-González HA, Zamorano-Jiménez CA. Estetoscopio, bata y corbata, y el riesgo de infecciones nosocomiales. Rev Invest Med Sur Mex. 2011; 18(4):195-202.

3. Lemus J. Manual de vigilancia epidemiológica; Serie HSP-UNI/ Manuales Operativos. Washington DC: OPS/ PALTEX; 1996.

4. Ministerio de Salud. Manual de vigilancia epidemiológica de las infecciones intrahospitalarias; Documento Técnico OGE-RENACE/ Vig. Hosp. DT 002-99 V1. Lima, Perú: MINSA/OGE; 1999.

5. Sehulster L1, Chinn RY; CDC; HICPAC. Guidelines for environmental infection control in health-care facilities. Recommendations of CDC and the Healthcare Infection Control Practices Advisory Committee (HICPAC). MMWR Recomm Rep. 2003; 52(RR-10):1-42.

6. Kramer A, Schwebke I, Kampf G. How long do nosocomial pathogens persist on inanimate surfaces? A systematic review. BMC Infectious Viseases. 2006; 6(1):130.

7. Bhalla A, Pultz NJ, Gries DM, et al. Acquisition of nosocomial pathogens on hands after contact with environmental surfaces near hospitalized patients. Infect Control Hosp Epidemiol. 2004; 25(2):164-7.

8. Hidron AI, Edwards JR, Patel J, and et al. NHSN annual update: antimicrobial-resistant pathogens associated with healthcare-associated infections: annual summary of data reported to the National Healthcare Safety Network at the Centers for Disease Control and Prevention, 2006-2007. Infect Control Hosp Epidemiol. 2008; 29(11):996-1011.

9. McDonald LC, Owings M, Jernigan DB. Clostridium difficile infection in patients discharged from US short-stay hospitals, 1996-2003. Emerg Infect Dis. 2006; 12(3):409-415.

10. Álvarez T, Herrera JF, Ávila-Agüero ML. Estetoscopio: Fuente potencial de infección nosocomial. Costa Rica, San José. Acta pediatr Costarr. 2005; 19(1): 8-12.

11. Mauriella MA, Pierson C, Chenoweth C. The stethoscope: a potential source of nosocomial infection. Arch Intern Med. 1997; 157(7):786-790.

12. Echevarría J. Estado actual de la resistencia bacteriana. Diagnóstico. 2008; 47(4):164-74.

13. Kennedy KJ, Dreimanis DE, Beckingham WD, Bowden FJ. Staphylococcus aureus and stethoscopes. Med J Aust. 2003; 178(9):468.

14. Merlin MA, Wong ML, Pryor PW, et al. Prevalence of methicillin-resistant Staphylococcus aureus on the stethoscopes of emergency medical services providers. Prehosp Emerg Care. 2009; 13(1):71-4.

15. Carey A, Saiman L, Polin R. Hospital-acquired infections in the NICU: epidemiology for the new millennium. Clin Perinatol. 2008; 35(1):223-49.

16. Souza M, Ueno M. Contaminação bacteriana de estetoscópios das unidades de pediatria em um hospital universitário. Rev Soc Bras Med Trop. 2009; 42(2):217-218.

17. Oliva-Menacho JE, García-Hjarles MA, Oliva Candela JA, De la Cruz-Roca HS. Contaminación con bacterias patógenas de estetoscopios del personal médico en un hospital de nivel III en Lima, Perú. Rev Med Hered. 2016; 27(2):83-88.

18. Kennedy KJ, Dreimanis DE, Beckingham WD, Bowden FJ. Staphylococcus aureus and stethoscopes. Med J Aust. 2003; 178(9):468.

19. Merlin MA, Wong ML, Pryor PW, et al. Prevalence of methicillin-resistant Staphylococcus aureus on the stethoscopes of emergency medical services providers. Prehosp Emerg Care. 2009; 13(1):71-4.

20. Souza M, Ueno M. Contaminação bacteriana de estetoscópios das unidades de pediatria em um hospital universitário. Rev Soc Bras Med Trop. 2009; 42(2):217-218.

21. Smith M, Mathewson J, Ulert A. Contaminated stethoscopes revisited. Archives of Internal Medicine.1996; 156(1): 82-84.

22. Zuliani ME, Maldonado AF, Bercial ME, Pedroso SA. Stethoscope: a friend or an enemy? Rev Paul Med. 2002; 120:13-15.

23. Kumarasamy KK, Toleman MA, Walsh TR, et al. Emergence of a new antibiotic resistance mechanism in India, Pakistan, and the UK: a molecular, biological, and epidemiological study. Lancet Infect Dis. 2010; 10(9):597-602.

24. Hernández M. Frecuencia de colonización de Staphylococcus aureus meticilino-resistente, de enterobacterias y de cándida spp. en estetoscopios y teléfonos móviles en una unidad de cuidados intensivos neonatal. Revista Salud Bosque. 2011; 1(1):17-24.

25. Brady R, Fraser S, Dunlop M, Paterson-Brown S, Gibb AP. Bacterial contamination of mobile communication devices in the operative environment. J Hosp Infect. 2007; 66(4):397-8.

26. Wood M, Lund R, Stevenson K. Bacterial contamination of stethoscopes with antimicrobial diaphragm covers. Am J Infect Control. 2007; 35(4):263-6.

Recibido: 05/12/2016

Aceptado: 29/08/2017 